\title{
Flavor Compounds in Wines Produced from Chardonnay Grapes Fermented with Fruit Juices
}

\author{
Sangeeta PATEL and Takayuki SHIBAMOTO* \\ Department of Environmental Toxicology, University of California, Davis, One Shields Avenue, Davis, CA 95616, USA
}

Received July 15, 2002; Accepted December 13, 2002

\begin{abstract}
Chardonnay grapes were fermented with or without juice of apple, pear, cantaloupe, peach, banana, or orange with a Saccharomyces cerevisiae yeast strain. Volatile chemicals formed in the seven wines were isolated and identified by gas chromatography/mass spectrometry. A total of 29 major volatile compounds were identified in the seven wines. They were 8 alcohols, 12 esters, 6 acids, and 3 miscellaneous compounds. Isoamyl alcohol was the most abundant volatile chemical found in all the wines. It comprised from $31.35 \%$ (pear wine) to $44.94 \%$ (orange wine) of the total GC peak area. Cantaloupe wine contained the highest amount of 2-phenyl alcohol in the seven wines. Amounts of monoethyl succinate, which was one of the most abundant compounds found in wine fermented alone, ranged from $8.35 \%$ (apple) to 23.95\% (banana). Ethyl 2-hydroxy propionate, amounts of which ranged from 3.93\% (cantaloupe) to 5.41\% (without fruit), was one of the major esters found in wines. The addition of fruit juices did not significantly change the overall volatile composition of fermented grapes. However, each fruit juice gave its characteristic flavor to the final wine. There have been only a few reports on wines flavored with other fruits.
\end{abstract}

Keywords: Chardonnay grapes, fruit juices, S. cerevisiae, flavor chemicals, wines

Although wine is the fermented product of grapes, various flavored wine beverages are also available. These flavored wines are prepared by adding flavoring materials, such as concentrated fruit essences, after the manufacture of the wine. Fruit flavored wines currently being produced include Wild Vines (E \& J Gallo), three fruit flavored wines under the Soleo brand (Sutter Home), and Arbor Mist (Canandaigua Wine Co.). Wine coolers, which are low alcohol wines flavored with fruit juices, have also been sold in the U.S.

There have been many reports on the volatile aroma compounds in fruit juices, including banana (Jordan et al., 2001), orange (Moshonas \& Shaw, 1994), peach (Horvat et al., 1990), apple (Lavilla et al., 1999), cantaloupe (Beaulieu \& Grimm, 2001), and pear (Takeoka et al., 1992). However, there have been virtually no reports on the volatile aroma compounds in fermented fruit juices other than grape juice. Studies on volatile aroma compounds in wine prepared from the fermentation of grapes and other fruits are also lacking. Consequently, it is unknown whether the aromatic qualities of non-grape fruits carry through the fermentation process. In the present study, volatile aroma compounds produced during fermentation of Chardonnay grapes with various fruit juices were investigated.

\section{Experimental}

Wine preparation Chardonnay grapes were harvested from the Sonoma Valley in September 1997. Grapes were crushed and maintained at temperatures of less than $36^{\circ} \mathrm{C}$ until transported to the Department of Environmental Toxicology, where the must was frozen at $-26.7^{\circ} \mathrm{C}$ for two weeks until ready

\footnotetext{
* To whom correspondence should be addressed.

E-mail: tshibamoto@ucdavis.edu
}

for fermentation. The must was fermented in 50-gal plastic containers fitted with a carbon dioxide vent through a water trap. Each batch of must was blended to 22 degree brix sugar measured with a Leica refractometer (Fisher Scientific, Pittsburgh, PA), and the titratable acidity ranged from 8.5-10 $\mathrm{g} / \mathrm{l}$. The sulfur dioxide concentration was adjusted to $50 \mathrm{ppm}$ using a $10 \%$ stock solution of potassium metabisulfite (Glen Ellen Winery, Modesto, CA). To each $2700 \mathrm{ml}$ of Chardonnay must, $300 \mathrm{ml}$ of various fruit juices (Fuji apple, pear, cantaloupe, peach, banana, and orange) were added. The juices of all fruits except orange were made by blending the fruit in a Waring blender and then straining out the pulp through a muslin cloth. Orange juice was squeezed using a Braun orange juice squeezer. Six 50-gal batches of must were each fermented with Saccharomyces cerevisiae Fermiblanc yeast (Gist Brocades, Canada). The amount of yeast added (22.7 $\mathrm{g} / 50 \mathrm{gal}$ ) was that recommended by the winemaker at the Glen Ellen Winery (Modesto, CA).

Fermentation was allowed to proceed at $16^{\circ} \mathrm{C}$ in a controlled temperature cooler for two weeks until the sugar concentration was reduced to less than $20 \mathrm{mg} / \mathrm{dl}$. The sugar was monitored using a glucose oxidase test strip (Chemstrip, Boehringer Mannheim Corp., Indianapolis, IN). The fermented must was racked three times: at 3 weeks, again 2 weeks later, and again after 2 months. The must was then filtered through cheesecloth into 750-ml wine bottles that were subsequently corked and stored in an $18^{\circ} \mathrm{C}$-storage chamber. The wines were stored at an ambient room temperature of $22^{\circ} \mathrm{C}$ for $1-3$ months. After one year, the wines were extracted and analyzed for volatile aroma compounds.

Isolation of volatile flavor compounds in wine A glass column $(250 \mathrm{~mm} \times 11 \mathrm{~mm}$ i.d.) packed with $5 \mathrm{ml}$ Porapak Q res- 
in (50-80 mesh, Alltech Associates, Inc., Deerfield, IL) was conditioned with 2 column volumes each of dichloromethane, methanol, and water. A 200-ml sample of wine was placed in the column connected to a 200-ml reservoir. The eluted wine was discarded. The column was further eluted with $50 \mathrm{ml}$ dichloromethane and the eluate was collected in a separatory funnel. A second volume of $10 \mathrm{ml}$ dichloromethane was added and eluted. A third 5-ml volume of dichloromethane was used to extract any remaining sample.

The water layer was discarded. The dichloromethane layer was transferred to an Erlenmeyer flask and dried over anhydrous sodium sulfate. After removal of anhydrous sodium sulfate, the sample solution was concentrated with a rotary evaporator and then further concentrated to a final volume of the sample of 1.2 $\mathrm{ml}$ with a purified nitrogen stream in a $5-\mathrm{ml}$ glass vial with a Teflon liner. The sample was stored at $-5^{\circ} \mathrm{C}$ until analysis for volatiles.

Determination of volatile compounds in wine The GC Kovats retention index I (Kovats, 1965) and the MS fragmentation pattern of each component were compared to those of the authentic compound to identify the volatiles in the samples. A Hewlett-Packard 5890 gas chromatograph equipped with a 30 $\mathrm{m} \times 0.25 \mathrm{~mm}$ i.d. DB-Wax bonded phase fused-silica capillary column (J \& W Scientific, Folsom, CA) and a flame-ionization detector (FID) was used for routine quantitative analysis. The split ratio was $1: 18$. The peaks from chromatography were integrated with a Spectra Physics 4290 integrator (San Jose, CA).
The injector and detector temperatures were maintained at $250^{\circ} \mathrm{C}$. The oven temperature was held at $50^{\circ} \mathrm{C}$ for $8 \mathrm{~min}$ and then programmed at $3^{\circ} \mathrm{C} / \mathrm{min}$ to $180^{\circ} \mathrm{C}$ for $35 \mathrm{~min}$. The linear helium gas flow rate was $30 \mathrm{~cm} / \mathrm{s}$.

A HP 5890A Series II GC interfaced to a HP 5972 massselective detector was used for mass spectral identification of the GC components at MS ionization voltage of $70 \mathrm{eV}$. Column and oven conditions were as stated above.

\section{Results and Discussion}

The Chardonnay grape originated in Lebanon and France. It became the only grape grown in Chablis, Burgundy (http: // wine.about.com/food/wine/library). The grape has also been used in sparkling wines and Champagne. The popularity of Chardonnay wine has grown immensely in the past forty years, and it is now the most popular white wine. Chardonnay was chosen for the present study because it possesses a wide variety of flavors.

The recovery method used for volatile compounds was one previously reported (Wada \& Shibamoto, 1997). The use of Porapak Q as a trapping agent and dichloromethane as a solvent has been shown to achieve satisfactory recovery of wine volatiles. For example, major wine volatiles such as 2-phenylethanol, diethyl succinate, and octanoic acid, could be nearly $100 \%$ recovered using this method (Wada \& Shibamoto, 1997). However, this method is unable to isolate semivolatile compounds such as tartaric acid and their esters. Table 1 shows the major vol-

Table 1. Volatile compounds found in chardonnay wine fermented with and without the addition of various fruit juices.

\begin{tabular}{|c|c|c|c|c|c|c|c|}
\hline \multirow{2}{*}{ Compound } & \multicolumn{7}{|c|}{ GC peak area $(\%)$} \\
\hline & No addition & Fuji apple & Pear & Cantaloupe & Peach & Banana & Orange \\
\hline \multicolumn{8}{|l|}{ Alcohols } \\
\hline 1-Propanol & 0.33 & - & 0.25 & 0.53 & 0.44 & 0.37 & 0.49 \\
\hline Isobutyl alcohol & 2.75 & 3.79 & 2.80 & 3.04 & 3.94 & 4.84 & 2.32 \\
\hline Isoamyl alcohol & 35.04 & 38.86 & 31.35 & 32.27 & 36.21 & 34.09 & 44.94 \\
\hline 1-Hexanol & 0.88 & 1.08 & 1.14 & 0.54 & 0.72 & 0.97 & 1.86 \\
\hline 2,3-Butanediol (D, L) & 3.04 & 2.63 & 2.03 & 2.30 & 3.67 & 2.50 & 3.16 \\
\hline 2,3-Butanediol (meso) & 1.33 & 0.81 & 0.81 & 0.77 & 1.09 & 0.31 & 0.79 \\
\hline Benzyl alcohol & 0.26 & - & - & 0.55 & - & 0.95 & - \\
\hline 2-Phenylethanol & 11.41 & 14.75 & 12.96 & 21.16 & 12.70 & 9.59 & 6.86 \\
\hline \multicolumn{8}{|l|}{ Esters } \\
\hline Isobutyl acetate & 0.23 & - & - & - & - & 0.22 & - \\
\hline Ethyl butanoate & 0.22 & - & 0.19 & - & 0.25 & - & - \\
\hline Isoamyl acetate & 4.24 & 3.06 & 2.55 & 2.91 & 1.63 & 2.46 & - \\
\hline Ethyl hexanoate & 0.51 & 0.51 & 0.41 & - & - & 0.57 & - \\
\hline Hexyl acetate & 0.17 & - & - & - & - & 0.19 & - \\
\hline Ethyl 2-hydroxy propionate & 5.41 & 5.08 & 3.97 & 3.93 & 4.47 & 4.91 & 5.32 \\
\hline Ethyl octanoate & 0.88 & 0.97 & 0.83 & 0.64 & 0.56 & 1.08 & - \\
\hline Ethyl decanoate & 0.38 & - & 0.36 & - & - & 0.50 & - \\
\hline Diethyl succinate & 0.82 & 0.73 & 2.66 & 1.82 & 1.50 & 0.67 & 3.73 \\
\hline 2-Phenylethyl acetate & 3.09 & 2.67 & 2.32 & 1.72 & 2.44 & 2.33 & - \\
\hline Hydroxy diethyl succinate & - & - & - & - & - & 0.21 & - \\
\hline Monoethyl succinate & 11.94 & 8.35 & 21.13 & 9.72 & 16.88 & 23.95 & 21.36 \\
\hline \multicolumn{8}{|l|}{ Acids } \\
\hline Acetic acid & 3.76 & 1.89 & 2.04 & 5.93 & 2.60 & 2.75 & 2.62 \\
\hline Butanoic acid & - & - & - & - & - & 0.19 & - \\
\hline Isoamyl acid & - & - & - & - & - & 0.89 & - \\
\hline Hexanoic acid & 2.28 & 2.47 & 2.21 & 2.27 & 1.57 & 2.75 & 0.79 \\
\hline Octanoic acid & 4.11 & 4.68 & 4.01 & 4.67 & 3.31 & 5.14 & 0.57 \\
\hline Decanoic acid & 1.99 & 2.09 & 1.77 & 1.75 & 1.58 & 2.40 & - \\
\hline \multicolumn{8}{|l|}{ Miscellaneous compounds } \\
\hline Benzaldehyde & - & - & - & - & - & 0.54 & - \\
\hline$\gamma$-Buryrolactone & 0.26 & 0.75 & 0.52 & - & - & 0.76 & 0.37 \\
\hline 3-(Methylthio)-1-propanol & 0.14 & - & - & - & - & 0.11 & - \\
\hline Total GC $\%$ of volatiles identified & 95.48 & 95.17 & 95.95 & 96.52 & 95.56 & 96.24 & 95.18 \\
\hline
\end{tabular}


atile aroma compounds found in Chardonnay wine fermented with or without fruit juices, along with their GC peak area\%. Values are the average of two duplicate experiments. A total of 29 major volatile compounds were identified in seven wines fermented with or without various fruit juices; however, the compounds with GC peak area\% less than 0.01 were not reported. The composition of the major volatiles found in the Chardonnay wine was consistent with that reported previously (Wada \& Shibamoto, 1997). Isoamyl alcohol was the most abundant volatile chemical, comprising $35.04 \%$ of the total volatile chemicals in the wine fermented alone. Overall, the addition of fruit juices did not significantly change the composition of volatile chemicals. However, the addition of various fruit juices subtly changed the composition of the volatile aroma compounds and flavor in the resulting wines. Even though the content of isoamyl alcohol in fresh orange juice is very low $(0.001-0.067 \%)$ (Moshonas \& Shaw, 1994), the relative amount of isoamyl alcohol increasedfrom $35.04 \%$ to $44.94 \%$ - considerably with the addition of orange juice to the wine. Isoamyl alcohol possesses a peculiar winey-brandy-like taste in proper dilution (Arctandar, 1969).

The amount of 2-phenylethanol was the second greatest after isoamyl alcohol among alcohols found in the seven wines, which ranged from $6.86 \%$ (orange) to $21.16 \%$ (cantaloupe). And despite the fact that cantaloupe juice contained a rather low level of 2-phenylethanol (Beaulieu \& Grimm, 2001), cantaloupe wine contained the highest amount of 2-phenylalcohol of the seven wines. Major cantaloupe volatile compounds, ethyl 2-methyl propionate and ethyl hexanoate (Beaulieu \& Grimm, 2001), were not found in the cantaloupe wine. The amount of 2,3-Butanediol ( $D, L$ and meso), which was the third highest amount among the alcohols, was the greatest in peach wine.

The amount of monoethyl succinate, one of the most abundant compounds found in wine fermented alone, ranged from $8.35 \%$ (apple) to $23.95 \%$ (banana). Banana wine contained a large amount of monoethyl succinate, but there is no report on this compound in banana essence or banana fruit (Jordan et al., 2001). Succinic acid is one of the major acids found in wine (Aragon et al., 1998); consequently, many esters of succinic acid, such as monoethyl succinate, diethyl succinate, and diethyl 2hydroxysuccinate, were found in the wines. Therefore, one may conclude that fruit juices may contain high levels of succinic acid because the amount of monoethyl succinate almost doubled in the wines fermented with all fruit juices except apple. Monoethyl succinate contributes an acidic taste. Many esters of succinic acids may be present in wines. These succinic acid esters have been used in various flavor compositions, such as imitation butter, rum, brandy, grape, and raspberry (Arctandar, 1969). Ethyl 2hydroxypropionate, amounts of which ranged from $3.93 \%$ (cantaloupe) to $5.41 \%$ (no addition), was one of the major esters found in wines. Ethyl 2-hydroxypropionate is an ethyl ester of lactic acid. It possesses mild, fruity-ethereal, and butter-like tastes in aqueous media and has been widely used in flavor compositions such as in imitation butter, butterscotch, grape, and strawberry (Arctander, 1969).

Apple essence had a higher proportion of ethyl esters of propionic acid and butylic acid (Lavilla et al., 1999). However, apple wine did not contain many detectable esters. The amount of octanoic acid, which ranges from $0.57 \%$ (orange) to $5.15 \%$ (banana), was the greatest among the six acids found in the seven wines. Orange wine seems low in acid content compared with the other wines. The addition of cantaloupe juice increased the relative amount of acetic acid from $3.76 \%$ to $5.93 \%$.

The banana wine had a strong note of banana. The chromatograms showed the presence of more complex flavor compounds, including many esters. Isoamy acetate and ethyl hexanoate are associated with a strong banana flavor (Arctandar, 1969). The results reflect the flavor notes detected in the wine.

\section{Conclusion}

The addition of fruit juices did not significantly change the overall volatile composition of fermented grapes. However, each fruit juice gave its characteristic flavor to the final wine. There have been only a few reports on wines flavored with other fruits. A Chinese patent describes the preparation of a fruit-grain wine that has a flavor of both fruit wine and the grain wine (Fuqiang, 2000). Another Japanese patent (Chikako, 1998) describes the production of a black tea-flavored sparkling wine or beer, containing lemon juice. In this preparation, the black tea leaves were added before filtering a grain mash or during the boiling of the mash, and lemon juice was added after completing the fermentation. Most fruit-flavored wines are produced by adding the fruit flavors after fermentation of the grape must. This study may be the first to investigate the addition of fruit juice to the grape must prior to fermentation.

\section{References}

Aragon, P., Atienza, J. and Climent, M.D. (1998). Influence of clarification, yeast type, and fermentation temperature on the organic acid and higher alcohols of Malaise and Muscatel wines. Am. J. Enol. Vitic., 49, 211-216.

Arctander, S. (1969). Perfume and Flavor Chemicals. Published by the author, Montclair, New Jersey.

Beaulieu, J.C. and Grimm, C.C. (2001). Identification of volatile compounds in cantaloupe at various developmental stages using solid phase microextraction. J. Agric. Food Chem., 49, 1345-1352.

Chikako, S. (1998). No title. Japanese patent JP10179119.

Fugiang, S. (2000). Fruit grain wine. Chinese patent CN1267717.

Horvat, R.J., Champman, Jr., G.W., Robertson, J.A., Meredith, F.I., Scorza, R., Callahan, A.M. and Morgens, P. (1990). Comparison of the volatile compounds from several commercial peach cultivars. $J$. Agric. Food Chem., 38, 234-237.

Jordan, M.J., Tandon, D., Shaw, P.E. and Goodner, K.L. (2001). Aromatic profile of aqueous banana essence and banana fruit by gas chromatography-mass spectrometry (GC-MS) and gas chromatography-olfactometry (GC-O). J. Agric. Food Chem., 49, 4813-4817.

Kovats, E. (1965). Gas chromatographic characterization of organic substances in the retention index system. Adv. Chromatogr., 1, 229247.

Lavilla, T., Puy, J., Lopez, M.L., Recasens, I. and Vendrell, M. (1999). Relationships between volatile production, fruit quality, and sensory evaluation in Granny Smith apples stored in different controlledatmosphere treatments by means of multivariate analysis. J. Agric. Food Chem., 47, 3791-3803.

Moshonas, M.G. and Shaw, P.E. (1994). Quantitative determination of 46 volatile constituents in fresh, unpasteurized orange juices using dynamic headspace gas chromatograpy. J. Agric. Food Chem., 42, $1525-1528$.

Takeoka, G.R., Buttery, R.G. and Flath, R.A. (1992). Volatile constituents of Asian pear (Pyrus serotina). J. Agric. Food Chem., 40, 1925-1929.

Wada, K. and Shibamoto, T. (1997). Isolation and identification of volatile compounds from a wine using solid phase extraction, gas chromatography, and gas chromatography/mass spectrometry. J. Agric. Food Chem., 45, 4362-4366. 